\title{
Delocalization of Sound Recording and the Development of Transnational Networks: Music Production in the Post- COVID Era
}

\author{
Marco Antonio Juan de Dios Cuartas \\ Complutense University of Madrid \\ mjuanded@ucm.es
}

\begin{abstract}
The recording studio traditionally constitutes a part of a network which helps to define the concept of the music scene. The digital revolution has caused the definitive uprooting of the physical device, providing a concept of space in which new creative scenarios are generated within a virtual community. The global pandemic has forced people to work "in the box" a term we associate with performing the mixing and mastering processes inside the computer and without the use of any external processing device, using network connections, even among the most sceptical professionals. This fact poses a paradigm shift in the relationships (both on a professional and human level) between producer, sound engineer, and artist, definitively modifying the traditional methods of music production. The main objective of this research is to analyse, using a non-experimental descriptive methodology based on non-participant systematic observation, the influence of the digital development of music production, and the resignification of the recording studio and the concept of scene from the new reality that is generated in the professional environment of music production by the impact of COVID-19.
\end{abstract}

KEYWORDS: music production, recording studio, music scene, virtual communities, Remote Music Collaboration Software, post-COVID era

\section{From the Physical to the Virtual Recording Studio: Music Production as an Immaterial Process}

The recording studio is consolidated as a creative space from the second half of the 20th century. Associated with large record companies, studios constitute a part of their business network, taking part in the process that goes from the selection of 
artists and songs to musical arrangements and the increasing role of the creative processes related to recording technology. The association of the recording studio with the label determines, in many cases, the concentration of large studios in big cities: the companies and their studios thus exercise a powerful attraction both at a national level (transfer of artists from small to big cities), as well as international (artists approach those which are considered as reference sound centres). Watson points out in this sense how recording studios "play a central role in creating the 'sound' of particular music scenes, and act as a focal point for networks of musicians and musical creativity" (2015: 1). The starting point is to understand the recording studio as part of a network, as another agent that helps to define the concept of scene. As Connell and Gibson (2003) point out, the initial stages of the music production process involve, in many cases, small-scale creativity where bands and composers create music in garages, recording studios, or local pubs.

The analysis of the change in the way in which musicians relate to the recording studio, the substitution of physical contact for virtual contact in interpersonal relationships, and how performance or composition goes from constituting a collective act under the same space to an individual physical action that faces the collectivity of the cloud, constitutes the main objective of this research. Ultimately, it is intended to demonstrate that the profound change produced from the COVID19 pandemic serves to consolidate a trend towards the virtualization of the recording space that had been developing for approximately a decade, and that raises an interesting debate from musicology related to nostalgia for the past of sound recording or the concept of scene associated with the delocalization of traditional recording centres and artistic networks that are generated through the internet. In this paper I examine "nostalgia for places", analysing the use that has been made by the audio industry of the "immersive term", and of "nostalgia for the process", focusing on the so-called Remote Music Collaboration Software (RMCS), which allows the creation of networks that transcend the individualistic character that is attributed to working with DAWs.

Within this context, I focus my analysis on two particularly relevant aspects of the technological evolution in music production: on the one hand, how the limitations of the spaces in which these home recordings take place entail a desire to represent the old architectural structures; this aspect is directly related to the virtualization and transformation of music production into an immersive experience. On the other hand, I find that the loss of the collective character of the recording within the home studio entails a longing for the artistic relationships that were produced within studio spaces in previous decades.

The development of individual projects that come to form scenes, that transcend the local through the massive distribution channels of the agents of power, represented by the large record companies, undergoes a deep methodological change in the digital age (1). Connell and Gibson (2003) use a dichotomy perfectly applicable to our case study: "fixity" and "fluidity" (2003: 9). "Fluidity" or "spatial mobility" indicate flows of people, goods and money through space. Music, as the authors point out, is both a merchandise and a cultural expression, and represents the most fluid of cultural forms. In the context of recording, music production represents precisely this balance between creation and distribution, between a work of art and a commercial product. The musical production developed in the environment of the recording studio generally has as its main motivation to transcend the local. In this sense, large cities also function as a centre of attraction for record production. Connell and Gibson associate the term "fixity" with the agglomeration trend at an industrial level. The recording industry has traditionally focused on big cities for a simple reason: these cities are where the headquarters of 
the major record companies are placed. During the analogue era, the proximity among the recording studio, the record factory and the subsequent physical distribution by companies was a fundamental element for business optimization. The activity of engineers and producers, directly connected to the big record companies, ended up defining a work style and, as a consequence, a sound that is geographically related. Large recording studios have exerted an important attraction for new talents who come to these centres to learn the job through senior engineers and producers that they consider their references: in this sense, tradition and learning based on oral transmission have been decisive to configure these "reference sound centres". But as Owsinski (2014) points out, there has been a homogenization of styles in recent years as a consequence of the mobility of sound engineers. Despite this, according to the author, we can still find four main styles inherited from the past: New York, Los Angeles, London, and Nashville (2).

This urban approach to recorded music has been explained by Leyshon (2001) through the concept of "musical network" where he identifies four main networks: creativity, reproduction, distribution, and consumption. Each of them plays a specific role in the music economy value chain and has its own distinctive geography. The relationship of recording studios with urban environments has also been studied by Gibson (2005) considering its characteristics as a space for interrelation among musicians, composers, producers and other agents of the music industry. In this way, we can relate the recording studio to a physical space as opposed to the immaterial evolution of music production processes in the digital age where, as we will see, immersion or emulation constitute its last stage.

Out of this concentration of the recording industry and its leading artists arose the separation between two markedly different traditions: the tradition of music production in the United Kingdom, and that in the United States. The comparison between the UK and US sound represents much more than two ways of methodologically approaching the recording and mixing processes. ZagorskiThomas (2012) assumes that certain technological, economic, and social factors have influenced the production practices of the two countries in different ways, although the personal reactions of professionals to these factors can vary considerably. Using the concept of centrifugal and centripetal forces in social and creative practice introduced by Bakhtin (1982), Zagorski-Thomas considers that for the individual professionals there are centripetal forces derived from their training or their socio-economic context, which entail a certain standardization, but they compete with the centrifugal forces derived from their own personalities.

The close relationship of recording studios with technological development, at the moment in which the recording studio was part of the same corporate structure as the record company, can also be considered a determining aspect to take into account. Some companies such as EMI had, from their origin in the 1930s, their own R\&D department, where the recording and processing devices were created and later used within the recording studio. This infrastructure leads audio professionals to associate the characteristics of a recorded sound with a geographical location, when they start from the consideration that it can only be generated in a specific acoustic space and with specific devices. The increase in independent recording studios during the 1970s coincided with a growing loss of interest on the part of large companies in internally managing technological development and the recording process. This fact gave way to a new era dominated by the large audio companies, whose potential clients were the new independent recording studios; companies such as Neve or Solid State Logic may have found a potential market in the emerging independent recording studios in which to locate their innovative products. As time went by, engineers, producers, and the artists 
themselves began to question the meaning of the vertical integration model of record companies where all decisions were imposed, advocating greater freedom in their projects, and also taking into account the strict rules that this studios system imposed regarding the correct use of the equipment, the strict schedules, and so on. In this way, independent studios began to appear that tried to cover this need for experimentation of artists. Some of these new studios, such as Trident Studios in London, had the budget and human resources to design their own equipment, while others had to hire specialized engineers. The studios no longer had an R\&D department to develop their devices, and they were forced to outsource this service.

This fact was decisive for the engineer Rupert Neve, who took advantage of this new situation to face the design of commissioned equipment for independent studios around the world. The American magazine $d B$ The Sound Engineering Magazine published the following information in February 1969:

The largest music-recording control console yet built by Rupert Neve and Company Limited, has been installed in the New York studios of the Vanguard Recording Society, Inc. The Cambridge, England-based firm's console embodies twenty-four input channels, sixteen output groups, four echo groups, and two foldback groups. The console also has comprehensive four-speaker monitoring and re-mix for sixteen tracks. This is their second installation for Vanguard, the first being a sixteen channel mastering console. Neve has recently completed two consoles installed in Spain and is currently working on units for re-recording desks for Pye Records, Ltd., and Associated British Pathe Ltd., both of England. (People, Places, Happenings 1969: 36)

The Neve factory in Cambridge or Solid State Logic in Oxford are consolidated as new "reference sound centres" in the new context of independent studios, and set the foundations of an important export industry to third countries, where an idealised sound from British recording studios is sought which is tried to be imitated in local record productions. Although the standardization of recording and mixing equipment in different countries could blur, a priori, the geographic reference associated with the sound of the recordings, this continued to take hold: owning Neve equipment in another country (as in the case of Spain) facilitated the approach to that British sound. It is important to highlight in this sense that the role of the audio engineer and the music producer arises in other countries from the imitation of the Anglo-Saxon model: to imitate their techniques, a main objective was to be able to count on the same equipment as far as possible.

Music production represents a unique professional field in terms of its technological development. Current music production continues to be deeply influenced by devices that are related to certain quality standards: microphones, preamps or mixers developed decades ago and that in today's digital context are still considered synonymous with quality. In a context in which the microphone as a transducer that converts acoustic waves into electrical signals would represent the only and necessary access route from the analogue to the digital world, and where computers have enough processing capacity to apply all the necessary audio processing in a musical production, hybrid systems have continued to be used maintaining the presence of classic analogue equipment considered by many professionals as synonymous with quality.

The consolidation of this equipment as a benchmark for professional audio has an inevitable consequence in the further development of the audio industry: tradition meets imitation. On the one hand, the spectacular economic development of China in the $21^{\text {st }}$ century caused the appearance of low-cost products that take 
models that we traditionally associate with English, German, or American brands as a reference. On the other hand, an imitation is developed through its virtualization by means of plugins that emulate the original devices. Technological development in the field of computer engineering has focused its efforts on reducing the differences between original and copy through techniques such as convolution or physical modelling. But despite the spectacular development of these technologies, a certain idealization towards the original physical device has continued to be maintained: the dream of a music producer in the 21 st century continues to be to have the devices of the 20th century, the physical LA2A (3) and not its emulation in plugin (4). The evolution of music recording and production digital technology during the first two decades of the 21 st century has focused much more on the imitation of analogue models than on the design of interfaces that distance themselves from their physical referents. Although this phenomenon could be associated with a business strategy to connect with a generation of engineers and/or producers who lived through the transition from the analogue to the digital era, the new generations demand, in the same way, the sound and controls of an original device that in most cases they have not seen or heard outside the computer.

Some authors have made sense of this process of mythologizing the past in terms of "technostalgia" (Pinch and Reineke 2009; van der Heijden 2015; Williams 2015). Williams centres his article on the manifestation of nostalgia around four areas: nostalgia for music, something that is part of our sense of musical history and that we must relate to the reaction to change; nostalgia for places: "the vicarious experience of greatness involves not only owning and operating the actual gear (or virtual facsimile), but also standing on the same linoleum [floor] as the greats of yesteryear" (2015); nostalgia for technology, which arises logically when we try to make great recordings like the ones that inspired us using the same machines that captured and shaped those sounds; nostalgia for the process, where the "collaborative achievement" of the past meets the individualistic tendency of working in the DAW. The individualistic nature of the home studio undergoes a progressive transformation with the creation of networks that allow sharing the same project through the cloud, generating a delocalization of sound that definitely culminates in the personal and professional relationships derived from the COVID19 pandemic and that poses profound changes in the way in which recorded music can be produced from now on.

\section{Delocalization of Sound Recording: Music Production as a Collaborative and Immersive Practice}

The development of DAW software during the second decade of the 21 st century has tried to fix the focus on the interconnection of different users through the internet. The so-called Remote Music Collaboration Software (RMCS) has normalized the human-machine interaction in the digital frontier, whereby the boundary between the digital-intangible and the analogue-physical worlds, appears increasingly blurred. This new reality, in which the recording studio is heading towards its definitive virtualization, raises new paradigms in the relationships between performers and creators, simultaneously developing projects that no longer share the same physical space: 
Digital processes continue to disperse the idea of the studio (and, indeed, the band), allowing musicians to collaborate without having to meet face to face and collapsing spatial distinctions between here and there, local and global, inside and outside. (Prior 2018: 83).

The impact of digital technology conditions the new working methods while facilitating the tools that make them possible. In the new technological and social context in which we live, the traditional processes of record production suffer a fragmentation and delocalization that disconnects them from a specific physical space. This new context not only implies a break with the roots of the physical by a generation of digital natives who have not experienced the transition from hardware device to software, it also implies a change in the very conception of music production, and in the way in which the necessary training to compete in a new job market is accessed. This new "culture of the virtual" establishes new working methods within some production phases that differ from the traditional ones, generating equally profound changes in the professional profiles and in the audio industry itself. In this new scenario, the use of Remote Music Collaboration Software (RMCS) creates new paradigms in the relationships between musicians and music producers, transforming the structures of social organization in which these creative projects have previously been developed: simultaneous management of the same project from different geographical locations, remote recording and mixing, creative negotiation through instant messaging (through the DAW itself or using WhatsApp audio notes, for example), etc.

The integration of social networks within these computer programs also increases the optimization and dissemination of the participants' work, providing an integrated view of creation and communication-management tools (video camera, chat, text boxes with annotations about the project, etc.), creating a virtual community that generates new models of musical production and dissemination with a significant cultural, social and economic impact. Renowned companies such as Avid or Steinberg, together with new online platforms such as Splice or Output, have opted for the development of tools that facilitate the recording and mixing process within the cloud, allowing the interaction with other users who share the same network. Behind the development of these applications there is a clear strategy aimed at creating community. The term community has served to explain the meaning of music in everyday contexts where the interaction among individuals allows us to recognize their geographical identity; it allows us to contextualize that physical interaction, but is equally applicable to a virtual interaction related to a physically dissociated space. These "symbolic anchors" referred to by Lewis (1992) allow people to articulate a sense of union closely related to the weight of tradition and the roots in the space of coexistence. But the community must be also understood as a "romantic construct" (Bennett 2004: 224): "as a means through which individuals who lack the commonality of shared local experience can cast music itself as a 'way of life' and a basis for community". Although the concept of community within music and its relationship with other concepts such as subculture or scene has already been treated by numerous authors (Frith 1981; Straw 1991; Cohen 1991; Bennett 1999; Connell and Gibson 2003) within a broader perspective that analyses the behaviour of audiences and their relationship with artists and musical genres, it could also be found that some of these concepts are equally applicable from the perspective of musical production, from the agents involved in the record creation process. In music production processes, we could understand the idea of community as a professional need. For example, the creative product is generated in many cases after multiple exchanges of information, 
experiential exchanges, trial and error tests with musicians, engineers and/or producers who contribute ideas that are rejected within a long negotiation process: arrangements that are discarded, takes that are discarded, tracks that are discarded and even complete productions that are discarded. The opinion "of the other" thus becomes something fundamental for the final configuration of the product. It is common to see in the credits of a mainstream record that the recording, mixing and mastering process includes studios and professionals located in different countries in the same work. The point I want to reach with my approach is that, in a vast majority of cases, the idea of community constitutes an intrinsic factor in the process of musical production where the "creative collective" replaces the "individual creator" (Hennion 1983). Obviously, in this approach we can identify exceptions; working with the DAW entails, de facto, an individual behaviour that we can associate with the practices of the electronic music producer, among others, but through this article I intend to defend the need that software developers have had to implement the ability to create community within the DAW. Although we cannot ignore the purely commercial intention of increasing engagement between producer (understood as client) and brand, I defend the idea that this strategy hides an inherent characteristic of the production practice: its collective character.

The Remote Music Collaboration Software (RMCS) ultimately allows one to record, edit and mix directly in the cloud by interacting with other people physically located in different places. Despite the technological milestone involved in sharing and following the same production project from anywhere in the world where you have an internet connection, this DAW is not normally presented as the virtual version of offline software on the computer, but rather as the opportunity to create community within a creative environment. There are many virtual platforms that compete to attract a generation of new producers who often interact through social networks and who consider these platforms as one more extension of their social life, such as through Instagram or Twitter. As an example, Avid Cloud Collaboration is linked to the so-called Artist Community, where musicians and producers share their demos in search of second and third opinions under the formula of what has been called mentoring, or offer their final results seeking patrons who finance the commercial projection of their work.

With the creation of these networks, the recording studio becomes a metainstrument (Moorfield 2006) at the service of musical creation in which creativity arises from collective interaction. The traditional recording studio, of large dimensions and with controlled acoustics, is no longer the objective of the big audio brands, which now focus on small clients who seek their motivation from the DIY philosophy and in the field of the home studio. The digital disruptive process entails, a priori, a loss of the recording studio's connection capacity with the rest of the agents that make up the traditional network of the music industry, and the studio ceases to be that reference space that we alluded to previously within the urban context to which the offices of the large multinational record companies belong. Hyper-connectivity in the network has had to face the great influence that tradition exerts even on new generations, although, far from constituting a problem, it has become the most powerful commercial strategy for brands.

\section{The Virtual Journey: Musical Production in the No-Place}

The final objective of my analysis is to demonstrate that this digital evolution in the audio industry serves to provide the necessary tools for the current context of a global pandemic, and that these tools definitely represent a paradigm shift in the 
dynamics of music creation and production. Like the different videoconferencing systems, these tools "were already there", in that they were technologically possible, but they remained in the background compared to traditional work methodologies in which presence continued to dominate the professional sector.

The replacement of a physical space by a virtual representation experienced through headphones has been brought about by different commercial products such as the one presented by Waves company in 2019: a plugin created in collaboration with Abbey Road Studios and with immersive Nx technology with the aim of providing a virtual journey from the home studio to the legendary control room of these recording studios. Regarding the launch, Mix magazine published the following review:

The Studio 3 control room is Abbey Road's flagship mix room, designed by the world's greatest acousticians to provide the ultimate music production and mixing environment. This unique control room is home to music's greatest legends. From modern classics by Radiohead, Amy Winehouse and Kanye West, to contemporary mixes of the Beatles and Pink Floyd, to number-one chart toppers by Frank Ocean, Brockhampton and Florence + the Machine: all were recorded, mixed and perfected at Abbey Road Studio 3 (Mixonline 2019).

The audio market already had a wide catalogue of acoustic environment emulation software using convolution reverbs, as well as acoustic space correction systems (ARC System by IK Multimedia, KRK Ergo) and emulation systems implemented for headphone-based monitoring devices (Focusrite VRM). Each and every one of these systems transforms real space into an immersive sound space, "a carefully and intuitively designed surrogate environment that creates a more fullbodied experience involving the senses to a fuller capacity than traditional media" (Droumeva 2005: 166). Beyond the possibilities of these technologies to turn listening into an immersive, interactive, and adaptive experience, the greatest potential that these tools seek to bring to their engineer-producer clients is that "virtual tourist experience" that is presented as a path towards the sound of legendary artists and albums, in short, a path to success. The comparison of the workspace with a unique and unrepeatable temple or sanctuary is recurrent in reviews in magazines and in promotional videos of this type of product. As we can hear in the voiceover of the promotional video of the Waves Abbey Road Studio 3 plugin:

It's about trusting the place where we create, our home, a sanctuary, our own personal shrine of sound. Now imagine your place can also be the best sounding room in the world: Imagine your place could be the legendary Abbey Road Studio 3. No matter where you really are. (Waves 2019a)

The idea of no-place subjects music production to a dissociation between creator and geographic location, and appears as a permanent slogan in different DAWbased production systems. But despite the fact that a slogan such as "Make Music Everywhere", used in the Avid Cloud Collaboration promotional campaign, is repeated as a mantra among the different manufacturers, the link with the recording studio from a traditional perspective is still present. In the words of Gregg Lehrman, creator of the online platform Output: "We are working to inspire a new generation of music makers with a platform that brings the power of a studio to anyone, wherever they are" (Gottsegen 2020). Once again, we are talking about an immersive practice, of the resignification of an idealised physical structure through 
several generations: a journey to/from the conventional recording studio, an exercise in abstraction from the no-place.

The experience is completed with the use of digital emulations of the physical devices that make up the unique character of those places: plugins that bring us the heat of the tubes, the harmonic distortion of the preamps, compressors or equalizers that contribute to the unique experience of going through these places. The account is sometimes completed with the testimonies of those who have been there and have used this equipment; that Alan Parsons confirms that the feeling is surprisingly similar (Waves 2019b) it is a determinate piece of information allowing us to believe that we can access the conditions that led to those works that we consider as references.

The relationship among the acoustics generated by the characteristics of the space, the location of the receiver, and the dialectical-musical discourse is close to the term "proxemics" coined by the American anthropologist Edward T. Hall (1966) to describe his theory of the nature of space constructed from both a cultural and a psychological perspective. The study of the way in which people occupy a space, and the distance that we keep among ourselves to communicate verbally - aspects that fall within what we call non-verbal communication - also finds its applicability in the virtual creation of spaces and the location of the different sound elements within them. In his analysis, Hall recognizes that there are cultural variations in the perception and interpretation of social space, and identifies four categories of space: intimate, personal, social, and public. Hall identifies various forms of meaning that can be associated with these proxemic categories, attributing national characteristics that describe the social constructions of levels and forms of proximity accepted in different cultures. Intimate space, as defined in Hall's proxemic categories, is associated with physical and emotional warmth, although intimacy can also be associated with honesty and sincerity. In this way, the use of the intimate space of the recording studio is capable of creating a metaphorical meaning that suggests a personal relationship with this space and its protagonists.

The relationship of the engineer-producer with the space, and with the material, is deeply modified as a consequence of this virtualization process, and it is one of the main causes of the professional sector's rejection of this type of technological innovation, hiding behind the debate between analogue and digital, and the widespread view that a digital version of the physical device always sounds worse. Rumsey, after an empirical analysis comparing the harmonic distortion generated by a tube amplifier and that recreated by means of a plug-in, considered that "although plug-ins may not have some of the raw physical appeal or nostalgic value of the classic hardware they emulate, they can bring the sounds of yesterday to a contemporary audience in a relatively convenient fashion" (2010: 427). In this sense, it seems that progress is being made gradually in changing the opinion that it is not a question of a better or worse sound, but a different one, and the obsession with the experience of using a physical device within the current digital context is losing strength. The last point we intend to reach is precisely how the pandemic situation has "forced" many engineers and producers to use these digital emulations, contributing to the conviction that we are inevitably facing a change of stage marked by the definitive uprooting of the physical device.

\section{Music Production in the Post-COVID Era}

The analysis of the evolution of music production technology and the methodological change that its virtualization implies leads to the main objective of 
this study: to analyse to what extent these music production methods of the postdigital era (Cascone 2004) have found their definitive foray into the professional sector in the context of the global pandemic generated by COVID-19.

On June 11,2020, a research conference on music production was organized at the Complutense University of Madrid in which five renowned engineers and music producers in Spain (Luca Petricca, Dany Richter, Javier Ortíz, Paco Martínez "Paco Loco" and Manuel Cabezalí) took part in a roundtable discussion that took place at a time when the recording studios were still closed or with strict security measures that drastically conditioned their working methods. The meeting took place taking into account their different approaches to the concept of contemporary recording studios: while some clung to the romantic idea of the classical studio, for the desire to own the original equipment, others saw work "in the box" as the solution to the feasibility of the studios as business models in the current context. The producer Luca Petricca pointed out within this debate:

I have mixed in analogue mixer format, analogue outboard and I am making the move to 'in the box'. It is a step that has given me a bit of life, more than anything else, I wish I could keep everything I had and continue to increase my equipment, but I am really adapting to this format. I've done a pretty interesting job with the 1176, for example, that we have in Reno [his recording studio] and with the 1176 from UAD [the plugin emulation] we did a comparison job, an exhaustive comparison in order to make the UAD sound as close to the 1176 as possible and we managed to bring it closer. They are not the same settings, but we managed to bring it closer. [...] We were all surprised, even engineer friends who didn't want it to look better, didn't want to see it, but even in a blind listening they couldn't tell the difference. (SonoLAB-UCM 2020) (5)

It seems increasingly difficult to defend from the professional sector the lower quality of the processes carried out within the computer. The arguments normally focus on defending the workflow with physical devices - a kind of performative act with knobs that is lost to the screen - or the sonic differences between original and copy where one does not sound worse than the other, but different. Once again, the listening of engineers and/or producers is conditioned by the spaces that they decide to revisit over and over again, recalling the sound of classic albums from the 1960 s and 1970s that they consider their references. The nostalgic approach to that "unlived" past and the feeling of vertigo towards the studio as a no-place justify, in many cases, these reluctances.

The disruptive digital process during the turn of the century caused the disappearance of a great majority of recording studios in the professional audio sector. These were large studios located in urban centres that, within the new music industry model, were no longer viable as businesses. Recording studios during the first two decades of the 21 st century have been built facing much more limited investments in smaller premises located on the outskirts of cities. This profound transformation has left behind a concept of the recording studio as a company that could only be possible with several partners contributing the necessary capital for the acquisition of extremely expensive equipment. The development of a more personal recording studio, the "producer's studio", managed to maintain the viability of the recording studio as a business and, more importantly, the professionalization of the sector outside of the amateur market that audio brands are strategically targeting. During this stage, as many home recording studios arise, in which young musicians, composers and producers dream of reaching the top, the professional sector also maintains its status: only in a professional studio can 
we find the original devices and, more importantly, the people who really know how to use them.

The current context of pandemic has not contributed anything new to the technological development of music production, but it has contributed to its use value. Remote collaboration tools, immersive acoustic experiences or emulations of physical devices through plugins were already there, but their use is beginning to be normalised within a professional sector where teleworking had never been an option. Traditionally, artists use the material and personal resources that are available to them in the artistic world in which they work, that is, the resources available make certain things possible, others easy and others difficult. Every pattern of availability "reflects the influences of a certain type of social organization and becomes part of the pattern of limitations and possibilities that makes up the art that is produced" (Becker 1982: 92). But the current professional context of recording studios, engineers and producers has forced artists to rethink their work methodologies, by creating situations in which physical presence is replaced by a "virtual visit" to the studios, or by a videoconference with the producer to decide the final takes of a song while the two of them visualize the same project on their computer screen.

The rise of virtual collaboration as part of the production process, blurring the line that has traditionally separated home recording from recording within a professional environment, is now beginning to be considered an acceptable fact, probably leaving as obsolete the debate that positions the idea of the democratization of access to recording against the deprofessionalization of the sector and the disappearance of the large recording studios. The really interesting debate for musicology thus focuses on the consequences of this new economic context for music creation. Although there are economic and social determinants that obviously influence the creative result of a production - record productions with the greatest capacity to influence engineers and producers at an international level have emerged in the First World, in cities with a dominant economic activity that has allowed the creation of technologically avant-garde infrastructures in an environment of cultural diversity (London, New York, Los Angeles) - in this new post-digital virtual context, creative individuals develop their work in a space whose cultural parameters also depend on the characteristics of the community they belong to within the network, a social environment that depends, in equal parts, on the physical location and the virtual relationship of its components.

The use of Remote Music Collaboration Software (RMCS) and plugins based on the idea of acoustic immersion and emulation of physical devices provides, on the one hand, interesting tools that benefit the exchange of ideas among the creative community, but on the other, it represents an important challenge for a professional sector where the action of the producer as an individual begins to exercise a more decisive role than ever over the product, a new scenario in which, taking up Bakhtin's concept, the centrifugal forces are imposed on the centripetal.

\section{Conclusion}

We will have to analyse the consequences of this change in the coming years, but it is clear that changes could be identified not only in the professional structures of the sector but also in record productions and the relationships of the artists within them. The British singer FKA Twigs recorded her last album in the context of a global pandemic during 2020, a work where all the collaborations were carried out telematically and where the entire production process was followed through video 
calls with the Spanish producer Pablo Díaz-Reixa "El Guincho". The development of this album, like many others that must be analysed in the future, is carried out in collaboration with musicians who have not met in person. The pandemic seems to have managed to finally incorporate in the professional sector work dynamics that were associated with an amateur musical practice related to a home studio. We are facing a transformation in music production processes as important as the appearance of multitrack tape recording or digital recording through a computer sound card may have been. As in all previous changes, multiple points of view are generated, conditioned by the generational link with the use of technology: what for one generation implies a profound transformation, for another represents a natural adaptation to the environment. The technological transformation of music production constantly generates that emotional dishonesty referred to by Frith (1988) and which is repeated every time a change shakes a labour system established for decades. Actually, the post-pandemic context will ultimately present new opportunities for the music industry. After all, resilience is a condition that has always marked this professional sector in its different disruptive transitions. But compared to previous transitions, there is a fundamental difference that makes this situation unique: it is a change in mentality, not a strictly technological change; the tools were already there, but they have now become completely necessary.

\section{Endnotes}

(1) Although some authors such as Hesmondhalgh (1997) already used the concept of democratization to analyse post-punk record labels in the 1980s, democratization appears as an inherent term in the digital disruptive process that occurs from the turn of the century.

(2) This localization of the recording in large cities could actually also be understood as a delocalization if we take into account the origin of the multitrack tape recording. It is worth remembering, in this sense, the contribution of key characters in the history of recording in the United States, such as the Allied Army soldier Jack Mullin or the guitarist Lester William Polsfuss (Les Paul). The fact that "sound labels" and recording studios such as Sun and Stax in Memphis, Muscle Shoals in Alabama, or Motown in Detroit are consolidated as "great centres" must be related not only to technological changes but also to different geopolitical changes that occur throughout history.

(3) LA2A refers to the legendary optical levelling amplifier.

(4) This is a generalized perception both among my music production students and the opinions of professionals. The idealization created around this audio equipment justifies the designs and the software manufacturers' business strategies.

(5) The 1176 refers to the original compressor designed by UA founder M.T. "Bill" Putnam.

\section{References}

\section{Bibliography}

Bakhtin, M.M. 1982. Dialogic Imagination: Four Essays. Austin, Tx: University of Texas Press.

Becker, H.S. 1982. Art Worlds. London: University of California Press.

Bennett, A. --

1999. Subcultures or Neo-Tribes? Rethinking the Relationship Between Youth, Style and Musical Taste. Sociology 33 (3): 599-617.

2004. Consolidating the Music Scenes Perspective. Poetics 32: 223-234. 
Cascone, K. 2004. The Aesthetics of Failure: "Post-Digital" Tendencies in Contemporary Computer Music. In C. Cox and D. Warner Eds. Audio Culture: Readings in Modern Music. New York: Bloomsbury Publishing: 392 - 398.

Castells, M. 2008. Communication Power. New York, NY: Oxford University Press.

Cohen, S. 1991. Rock Culture in Liverpool: Popular Music in the Making. Oxford: Clarendon Press.

Connell, J. and Gibson, C. 2003. Sound Tracks: Popular Music, Identity and Place. London; New York: Routledge.

dB The Sound Engineering Magazine 1969. People, Places, Happenings, 3 (2) February, p.36.

Droumeva, M. 2005. Understanding Immersive Audio: A Historical and SocioCultural Exploration of Auditory Displays. ICAD 05 - Eleventh Meeting of the International Conference of Auditory Display, Limerick, Ireland: 162 - 168.

Frith, S. -

1981. "The Magic that can Set you Free": The Ideology of Folk and the Myth of Rock. Popular Music 1: 159-168.

1986. Art versus Technology: The Strange Case of Popular Music. Media,

Culture and Society, Vol. 8. London: 263-279

Gibson, C. 2005. Recording Studios: Relational Spaces of Creativity in the City. Built Environment 31 (3): 192 - 207.

Gottsegen, G. 2020. Music Production Software Company Output Announces \$45M Series A. [online] Built In Los Angeles. Available at: https://www.builtinla.com/2020/10/21/output-raises-45m-series-a [Accessed 20 December 2020].

Hall, E.T. 1966. The Hidden Dimension. London: Doubleday.

Hennion, A. 1983. The Production of Success: An Anti-Musicology of the Pop Song. Popular Music 3: 159-193.

Hesmondhalgh, D. 1997. Post-Punk's Attempt to Democratise the Music Industry: The Success and Failure of Rough Trade. Popular Music 16 (3): 255 - 274.

Jenkins, H. 2006. Convergence Culture: Where Old and New Media Collide. New York, NY: New York University Press.

Lewis G. H. 1992. Who Do You Love?: The Dimensions of Musical Taste. In J. Lull Ed. Sage Focus Editions: Vol. 89. Popular Music and Communication. London: Sage: 134-151.

Leyshon, A. 2001. Time-Space (and Digital) Compression: Software Formats, Music Networks, and the Reorganisation of the Music Industry. Environment and Planning A: Economy and Space 33 (1): 49-77.

Moorfield, V. 2005. The Producer as Composer: Shaping the Sounds of Popular Music. Cambridge, MA: MIT Press.

Owsinski, B. 2014. The Mixing Engineer's Handbook. Boston: Thomson Course Technology.

Pinch, T. and Reinecke, D. 2009. Technostalgia: How Old Gear Lives on in New Music. In Bijsterveld, K. and van Dijck, J. Eds. Sound Souvenirs: Audio Technologies, Memory and Cultural Practices. Amsterdam: Amsterdam University Press: $152-166$.

Prior, N. 2018. Popular Music, Digital Technology and Society. London: Sage Publications Ltd. 
Rumsey, F. 2010. Digital Audio Effects and Simulations. Journal of Audio Engineering Society Vol. 58, No. 5: 420-427.

Straw, W. 1991. Systems of Articulation, Logics of Change: Communities and Scenes in Popular Music. Cultural Studies 5 (3): 368 - 388.

van der Heijden, T. 2015. Technostalgia of the Present: From Technologies of Memory to a Memory of Technologies. NECSUS. European Journal of Media Studies 4 (2): 103 - 121.

Watson, A. 2015. Cultural Production in and Beyond the Recording Studio. New York: Routledge.

Williams, A. 2015. Technostalgia and the Cry of the Lonely Recordist. Journal on the Art of Record Production 9. Available at: https://www.arpjournal.com/ asarpwp/technostalgia-and-the-cry-of-the-lonely-recordist/ Accessed 6 August 2021.

Zagorski-Thomas, S. 2012. The US vs the UK Sound: Meaning in Music Production in the 1970s. In Zagorski-Thomas, S. and Frith, S. Eds. The Art of Record Production: An Introductory Reader to a New Academic Field. Farnham: Ashgate Publishing Limited: 57-76.

\section{Videography}

Williams, A. 2015. Technostalgia and the Cry of the Lonely Recordist. Journal on the Art of Record Production 9. Available at: https://www.arpjournal.com/ asarpwp/technostalgia-and-the-cry-of-the-lonely-recordist/ Accessed 6 August 2021.

SonoLAB-UCM 2020. Mesa Audioforo "Paradigmas de la producción musical actual". [video] Available at: https://youtu.be/VnocsrOqNQs [Accessed 19 December 2020].

Waves, --

2019a. Introducing the Waves Abbey Road Studio 3 Plugin. [video] Available at: https://youtu.be/r7uEcub9QfY [Accessed 18 December 2020]. 2019b. Alan Parsons - First Reaction to the Abbey Road Studio 3 Plugin. [video] Available at: https://youtu.be/qaw-IKqXv0E [Accessed 18 December 2020].

\section{Web Sources}

Mixonline. 2019. Waves Audio and Abbey Road Studios Introduce the Abbey Road Studio 3 Plugin, Powered by Waves Nx Technology. [online] Available at: https://www.mixonline.com/the-wire/waves-abbey-road-studio-3 [Accessed 21 December 2020]. 